\title{
MOBILIDADE DO BORO EM PLANTAS DE ABACAXI ${ }^{1}$
}

\author{
SUSANA CRISTINE SIEBENEICHLER ${ }^{2}$, PEDRO HENRIQUE MONNERAT ${ }^{3}$, ALMY JUNIOR CORDEIRO DE \\ CARVALHO $^{4}$, JOSÉ ACCÁCIO DA SILVA ${ }^{5}$, AMANDA OLIVEIRA MARTINS ${ }^{6}$
}

\begin{abstract}
RESUMO - Para confirmar a mobilidade do boro em abacaxizeiro, cultivaram-se doze mudas da cultivar 'Pérola' em solução nutritiva completa, contendo $1 \mu \mathrm{mol} \mathrm{L}{ }^{-1}$ de B, em delineamento inteiramente casualizado. Após 45 dias, coletaram-se quatro plantas $\left(\mathrm{T}_{0}\right)$ e, nas oito plantas restantes, aplicou-se, por 3 dias consecutivos, uma solução de $\mathrm{H}_{3} \mathrm{BO}_{3}$ a $10 \mathrm{mmol} \mathrm{L}^{-1}$, pincelando-se ambas as faces da folha basal número sete (7). A partir do início da aplicação foliar, foi suspenso o fornecimento de B na solução nutritiva. Um dia após a terceira aplicação foliar, coletaram-se quatro plantas $\left(\mathrm{T}_{1}\right)$ e, 60 dias após esta, coletaram-se as últimas quatro plantas $\left(\mathrm{T}_{2}\right)$. As plantas foram fracionadas em diferentes partes nas quais o teor de $\mathrm{B}$ total foi determinado pelo método da azometina-H. Em $\mathrm{T}_{0}$, o maior teor de $\mathrm{B}$ observado foi na raiz; no $\mathrm{T}_{1}$, na folha pincelada e na raiz e, no $\mathrm{T}_{2}$, na folha pincelada. O conteúdo de $\mathrm{B}$ acompanhou a variação da massa seca das porções da planta, sendo que, no $\mathrm{T}_{2}$, o maior conteúdo de $\mathrm{B}$ e massa seca foram observados nas folhas novas formadas entre o $\mathrm{T}_{1}$ e $\mathrm{o} \mathrm{T}_{2}$. O boro contido nessas folhas $(29,8 \%$ do boro total da planta) proveio das folhas mais velhas (FMed, FTrat e FBas), cujo conteúdo diminuiu significativamente, confirmando a mobilidade do boro em plantas de abacaxi 'Pérola'.

Termos para indexação: Annanas comosus, remobilização de B, conteúdo de B.
\end{abstract}

\section{BORON MOBILITY IN PINEAPPLE}

\begin{abstract}
To confirm the mobility of boron in pineapple, twelve 'Pérola' pineapple seedlings were cultivated in a complete nutrient solution containing $1 \mathrm{mmol} \mathrm{L}^{-1} \mathrm{~B}$ in a completely randomized design. After 45 days, B was removed from the nutrient solution, and four plants were harvested $\left(\mathrm{T}_{0}\right)$, and in the eight remaining plants $10 \mathrm{mmol} \mathrm{L}^{-1} \mathrm{H}_{3} \mathrm{BO}_{3}$ solution were painted, for 3 consecutive days, on both faces of the basal leaf number seven (7). One day after the third foliar application, four plants were harvested $\left(T_{1}\right)$ and, sixty days later, the last four plants were harvested $\left(T_{2}\right)$. The plants were partitioned into different parts in which total $\mathrm{B}$ concentration was determined by azometina- $\mathrm{H}$ method. In $\mathrm{T}_{0}$ the largest $\mathrm{B}$ concentration was in the root; in $\mathrm{T}_{1}$, in the painted leaf and the root and, in $\mathrm{T}_{2}$, in the painted leaf. The content of $\mathrm{B}$ followed the variation of the dry weight of the portions of the plant, and in $\mathrm{T}_{2}$ the largest content of $\mathrm{B}$ and dry weight were observed in the new leaves formed between $\mathrm{T}_{1}$ and $\mathrm{T}_{2}$. Boron contained in those leaves $(29,8 \%$ of the total boron of the plant) came from the oldest leaves which content decreased significantly, confirming the mobility of Boron in pineapple plants.
\end{abstract}

Index terms: Annanas comosus, B mobility, B content.

\section{INTRODUÇÃO}

O micronutriente B tem sido considerado imóvel nas plantas em geral, por muitos anos; entretanto, estudos realizados, principalmente a partir da década de 80 , demonstraram que esta afirmativa não devia ser generalizada, pois verificou-se que este micronutriente é móvel em algumas espécies de plantas, tais como: macieira, ameixeira, cerejeira (Brown e Hu, 1998) e brócolis (Shelp, 1988).

Segundo estes estudos, a mobilidade do B é possível por este elemento se ligar a compostos que apresentam a configuração cis-diol. Estes compostos são itóis (álcoois de açúcar) como: sorbitol (macieira), manitol (brócolis) e dulcitol (Dordas et al., 2001). Os itóis são considerados compostos de estoque formados durante o processo fotossintético e transportados pelo floema em plantas superiores, sendo utilizados na nutrição heterotrófica e na osmorregulação (Loescher et al., 1995). O B se moveria, portanto, ligado a esses itóis.

A deficiência de $\mathrm{B}$ em plantas que não remobilizam este nutriente, apresenta-se nas suas porções novas. Em espécies muito exigentes em B, como o girassol, pode ocorrer a formação de folhas novas deformadas e a morte do meristema apical (Bergmann, 1986). Já plantas que transportam esses itóis no floema, apresentariam sintomas de deficiência de $\mathrm{B}$ nas partes mais velhas e, só em casos extremos, o sintoma seria observado em porções jovens.

$\mathrm{O} \mathrm{B}$, como os demais micronutrientes, é indispensável para as plantas, pois tem efeito direto na formação de novos tecidos e na sua produtividade, tornando-se igualmente importante conhecer se a sua redistribuição ocorre dentro da planta e se há variação entre as espécies (van Goor e van Lune, 1980).

O conhecimento da mobilidade dos nutrientes na planta favorece a escolha do tipo de manejo que será adotado na correção ou prevenção da deficiência. Quando o nutriente é imóvel na planta, tornase necessário o fornecimento direto nos novos órgãos em formação; entretanto, para elementos móveis, este tipo de manejo é desnecessário ou, então, a forma de aplicação pode ser facilitada.

A remobilização do B ocorre, principalmente, em plantas que são cultivadas em ambientes com baixa disponibilidade de B para a planta. Em solos com alto teor de B, o transporte deste nutriente ocorre, mormente, pelo fluxo transpiratório, e sua acumulação ocorre nos órgãos que apresentam a maior taxa transpiratória (Brown e Shelp, 1997).

A demora na manifestação do sintoma de deficiência de B em plantas de abacaxi cultivadas sem a adição de B, em solução nutritiva, sugeriu a possibilidade de esta espécie remobilizar o B (Siebeneichler, 2002), como ocorre em outras espécies. O presente ensaio foi conduzido com o objetivo de comprovar a mobilidade do B no abacaxizeiro 'Pérola'.

\section{MATERIALEMÉTODOS}

Este experimento foi conduzido em casa de vegetação do Setor de Nutrição Mineral de Plantas, do Laboratório de Fitotecnia, Centro de Ciências e Tecnologias Agropecuárias da Universidade Estadual do Norte Fluminense Darcy Ribeiro, Campos dos Goytacazes-RJ.

Doze mudas de abacaxi (Ananas comosus L.) da cultivar Pérola, tipo filhote, com aproximadamente $250 \mathrm{~g}$, foram cultivadas em solução nutritiva contendo os seguintes sais, em mmol L-1 : $0,7 \mathrm{~K}_{2} \mathrm{SO}_{4} ; 0,1 \mathrm{KCl}$; $2,0 \mathrm{Ca}\left(\mathrm{NO}_{3}\right)_{2} ; 0,5 \mathrm{Mg} \mathrm{SO}_{4} ; 0,1 \mathrm{KH}_{2} \mathrm{PO}_{4} ; 10^{-3} \mathrm{H}_{3} \mathrm{BO}_{3} ; 0,5.10^{-3} \mathrm{MnSO}_{4}$; $0,5.10^{-3} \mathrm{ZnSO}_{4} ; 0,2.10^{-3} \mathrm{CuSO}_{4} ; 0,01.10^{-3}\left(\mathrm{NH}_{4}\right)_{6} \mathrm{Mo}_{7} \mathrm{O}_{24} ; 0,04 \mathrm{Na} \mathrm{Fe}$ (III)EDTA; $\mathrm{pH} 5,5$. O ajuste do $\mathrm{pH}$ foi feito a cada dois dias com KOH $1 \mathrm{M}$ ou $\mathrm{HNO}_{3}+\mathrm{H}_{3} \mathrm{PO}_{4}$ a $5 \%$. A troca da solução foi realizada a cada 21 dias.

As mudas foram distribuídas em três caixas de madeira medindo $100 \times 50 \times 10 \mathrm{~cm}$, revestidas com plástico, contendo $40 \mathrm{~L}$ de solução

\footnotetext{
(Trabalho 157/2003). Recebido: 17/10/2003. Aceito para publicação: 23/06/2005.

${ }^{2}$ Eng $^{\mathrm{a}}$. Agr ${ }^{\mathrm{a}}$, Profa. Adjunta, Curso de Agronomia, Campus Universitário de Gurupi / Fundação Universidade Federal do Tocantins. Cx. P.: 66-CEP: 77.402-970/ Gurupi-TO. E-mail: susana@uft.edu.br;

${ }^{3,4}$ Eng $^{\mathrm{o}} \mathrm{Agr}^{\mathrm{o}}$, DSc. Professores, UENF/CCTA/LFIT, Av. Alberto Lamego, 2000, Horto, 28015-620, Campos dos Goytacazes - RJ. E-mail: monnerat@uenf.br;

${ }^{5}$ Eng $^{\circ}$ Químico, Téc. de Nível Sup., UENF/CCTA/LFIT, Av. Alberto Lamego, 2000, Horto, 28015-620, Campos dos Goytacazes - RJ;

${ }^{6}$ Estudante do Curso de Agronomia e bolsista do LFIT, UENF.
} 
nutritiva cada uma. As mudas foram fixadas em uma chapa de isopor com $2 \mathrm{~cm}$ de espessura, espaçadas de $30 \mathrm{~cm}$ e arejadas continuamente com uma bomba para arejamento de aquário. Essas caixas foram mantidas sobre uma mesma bancada, em casa de vegetação, com temperatura média das máximas e das mínimas de 45 e $24^{\circ} \mathrm{C}$, respectivamente.

O transplante das mudas para a solução nutritiva foi realizado no dia 11-02-02 e, nessa solução, com baixa concentração de boro, as plantas permaneceram por 45 dias, para que o teor de boro em seus tecidos ficasse baixo. A solução nutritiva foi, então, trocada por outra, sem boro, nas três caixas, quando, então, quatro plantas foram colhidas, ao acaso, para a caracterização do crescimento e do teor e conteúdo inicial de boro em diversas partes da planta. Essas plantas constituíram o tempo zero $\left(\mathrm{T}_{0}\right)$. Nas oito plantas que restaram, aplicou-se uma solução de $\mathrm{H}_{3} \mathrm{BO}_{3}$ a $10 \mathrm{mmol} \mathrm{L}^{-1}$ na folha número 7 , a contar da base. A aplicação de $\mathrm{B}$ foi realizada por 3 dias consecutivos, uma vez por dia, com um pequeno pincel, pincelando-se as porções abaxial e adaxial da folha, evitando-se o escorrimento da solução para a axila das folhas. Um dia após a última aplicação foliar, quatro plantas foram colhidas e constituíram o tempo um $\left(\mathrm{T}_{1}\right)$; sessenta dias após essa coleta, as últimas quatro plantas foram colhidas, constituindo o tempo $2\left(\mathrm{~T}_{2}\right)$. Os três tratamentos tiveram, portanto, quatro repetições em delineamento inteiramente casualizado.

Antes de colocar as plantas na solução sem B, o sistema radicular foi lavado em água deionizada corrente por 10 minutos.

As plantas de todos os tratamentos, após a colheita, foram secionadas em folhas basais (FBas: folhas 1 a 6 ), folha tratada (FTrat: folha 7), folhas medianas (FMed: 8 a 11), folhas superiores ou (FSup: 12 a 16) folhas novas, com menos de $10 \mathrm{~cm}$ de comprimento (FNov), caule (Caule) e raiz (Raiz). No $\mathrm{T}_{2}$, as folhas que se formaram durante os sessenta dias foram denominadas FNov1 (17 a 20), FNov2 (21 a 23), FNov3 (24 a 26) e FNov4 (folhas com menos de $10 \mathrm{~cm}$ de comprimento). As folhas basais, a tratada e as medianas foram consideradas folhas velhas no final do experimento, e as formadas durante os dois meses de cultivo sem boro foram consideradas folhas novas. As folhas foram limpas com algodão umedecido em água desionizada e as que receberam a aplicação de B foram lavadas em água desionizada corrente por 30 segundos.

A seguir, as partes das plantas foram secas em estufa de circulação de ar forçada, a $70^{\circ} \mathrm{C}$, por 72 horas. Após secas, as amostras foram pesadas e moídas em moinho tipo Wiley, passadas em peneira de
20 mesh e armazenadas em frascos hermeticamente vedados.

A metodologia utilizada na determinação do B consistia em pesar 250mg de massa seca (MS) que foram incineradas em mufla a $550^{\circ} \mathrm{C}$, por 4 horas. No dia seguinte, as cinzas foram retiradas da estufa, adicionados 3 a 4 gotas de peróxido de hidrogênio $\left(\mathrm{H}_{2} \mathrm{O}_{2}\right) 3 \%$, ressecadas em chapa quente e requeimadas por 3 horas a $550^{\circ} \mathrm{C}$. Após a segunda queima, as cinzas foram suspensas com $10 \mathrm{ml}$ de $\mathrm{HNO}_{3}$ 1:60. O restante da metodologia foi a preconizada por Malavolta et al. (1997), com a quantificação colorimétrica do $\mathrm{B}$ com o uso de Azometina $\mathrm{H}$.

Os dados foram submetidos à análise de variância, e as médias foram comparadas pelo teste de Tukey, ao nível de $5 \%$ de probabilidade. As análises estatísticas foram realizadas utilizando-se do programa SAEG.

\section{RESULTADOS E DISCUSSÃO}

As mudas, ao serem transferidas para a solução nutritiva, estavam com o teor de B relativamente baixo (menor que $10 \mathrm{mg} \mathrm{kg}^{-1} \mathrm{de}$ $\mathrm{B}$ na matéria seca foliar), mas as plantas não apresentavam sintoma visual de deficiência tanto na parte aérea como nas raízes.

Em espécies que não remobilizam o $\mathrm{B}$, como o girassol, o teor de $10 \mathrm{mg} \mathrm{kg}^{-1}$ promove sintomas nítidos de deficiência deste elemento nas porções novas da planta - folhas novas e meristemas (Bergmann, 1986).

Durante a condução do experimento, as plantas do tratamento $\mathrm{T}_{2}$ formaram, em média, nove folhas novas (folhas 18 a 26), causando aumento da massa seca na porção FNov (Tabela 1). Observou-se, também, aumento significativo da massa seca nas porções FSup (folhas 12 a 16) e na Raiz, e não se observaram sintomas visuais da deficiência de $\mathrm{B}$ em nenhuma das plantas.

Quanto ao teor (Tabela 1), observou-se que, no $\mathrm{T}_{0}$, o maior teor de B foi observado na raiz. Após a aplicação foliar de B $\left(\mathrm{T}_{1}\right)$, as porções FTrat e a Raiz apresentaram os maiores teores, não diferindo entre si.

Ao comparar os teores de B, nas diferentes partes da planta, no $T_{0}$ e no $T_{1}$, observa-se que este teor aumentou significativamente, nas porções FSup, FTrat e na FBas (Tabela 1). Este incremento pode ser atribuído ao $\mathrm{B}(10 \mathrm{mM})$ aplicado na folha 7 , que se acumulou na própria (FTrat) e também se translocou para as folhas abaixo (FBas) e acima dela (FSup).

TABELA 1 - Massa seca e teor, conteúdo e distribuição de Boro em diferentes partes das mudas de abacaxi 'Pérola' colhidas em 3 épocas.

\begin{tabular}{|c|c|c|c|c|c|c|c|c|}
\hline \multirow[t]{3}{*}{ Tratamento ${ }^{\prime \prime}$} & \multicolumn{8}{|c|}{ Partes da planta ${ }^{2}$} \\
\hline & $\begin{array}{c}\text { FNov } \\
(17 \text { a 26) }\end{array}$ & $\begin{array}{c}\text { FSup } \\
(12 \text { a 16) }\end{array}$ & $\begin{array}{c}\text { FMed } \\
(8 \text { a11 })\end{array}$ & $\begin{array}{l}\text { FTrat }^{3^{/ /}} \\
\text {(folha 7) }\end{array}$ & $\begin{array}{c}\text { FBas } \\
\left(\begin{array}{l}1 \\
\text { a }\end{array}\right)\end{array}$ & Caule & Raiz & Total \\
\hline & \multicolumn{8}{|c|}{ Massa Seca (g) } \\
\hline T0 & $0,30 \mathrm{bB}$ & $2,38 \mathrm{bB}$ & $6,20 \mathrm{aA}$ & $1,59 \mathrm{bA}$ & $6,63 \mathrm{aA}$ & $1,14 \mathrm{bA}$ & $0,65 \mathrm{bB}$ & $18,9 \mathrm{~B}$ \\
\hline T1 & $0,40 \mathrm{bB}$ & $2,75 \mathrm{bB}$ & $7,83 \mathrm{aA}$ & $1,64 \mathrm{bA}$ & $6,53 \mathrm{aA}$ & $1,54 \mathrm{bA}$ & $0,74 \mathrm{bB}$ & $21,4 \mathrm{~B}$ \\
\hline \multirow[t]{2}{*}{ T2 } & $11,01 \mathrm{aA}$ & $4,87 \mathrm{bcA}$ & $6,85 \mathrm{bA}$ & $1,42 \mathrm{dA}$ & $7,01 \mathrm{bA}$ & $2,40 \mathrm{dA}$ & $3,24 \mathrm{cdA}$ & $36,8 \mathrm{~A}$ \\
\hline & \multicolumn{8}{|c|}{ Teor de Boro $\left(\mathrm{mg} \mathrm{kg}^{-1}\right)$} \\
\hline T0 & $9,20 \mathrm{bA}$ & $7,85 \mathrm{bcB}$ & $6,98 \mathrm{bcAB}$ & $6,25 \mathrm{bcB}$ & $5,47 \mathrm{cB}$ & $7,21 \mathrm{bcA}$ & $12,80 \mathrm{aA}$ & \\
\hline T1 & $9,61 \mathrm{bcA}$ & $10,32 \mathrm{bA}$ & $8,83 \mathrm{bcA}$ & $16,02 \mathrm{aA}$ & $10,12 \mathrm{bcA}$ & $7,18 \mathrm{cA}$ & $14,94 \mathrm{aA}$ & \\
\hline \multirow[t]{2}{*}{$\mathbf{T 2}$} & $5,43 \mathrm{abB}$ & $5,15 \mathrm{bC}$ & $4,83 \mathrm{bB}$ & $8,39 \mathrm{aB}$ & $5,09 \mathrm{bB}$ & $5,76 \mathrm{abA}$ & $5,50 \mathrm{abB}$ & \\
\hline & \multicolumn{8}{|c|}{ Conteúdo de Boro ( g) } \\
\hline T0 & $2,72 \mathrm{cB}$ & $18,94 \mathrm{bcA}$ & $43,70 \mathrm{aB}$ & $10,24 \mathrm{cB}$ & $37,27 \mathrm{abB}$ & $8,19 \mathrm{cA}$ & $8,30 \mathrm{cA}$ & $129,4 \mathrm{~B}$ \\
\hline T1 & $3,78 \mathrm{cB}$ & $28,26 \mathrm{bA}$ & $69,63 \mathrm{aA}$ & $26,75 \mathrm{bA}$ & $65,27 \mathrm{aA}$ & $11,07 \mathrm{bcA}$ & $10,57 \mathrm{bcA}$ & $215,3 \mathrm{~A}$ \\
\hline \multirow[t]{2}{*}{$\mathbf{T} 2$} & $59,93 \mathrm{aA}$ & $25,57 \mathrm{bA}$ & $34,90 \mathrm{bB}$ & $11,97 \mathrm{cAB}$ & $35,73 \mathrm{bB}$ & $13,84 \mathrm{cA}$ & $17,85 \mathrm{bcA}$ & $199,8 \mathrm{~A}$ \\
\hline & \multicolumn{8}{|c|}{ Distribuição do Boro $(\%)^{4 /}$} \\
\hline T0 & $2,1 \mathrm{cB}$ & $15,1 \mathrm{bA}$ & 33,8 aA & $7,6 \mathrm{cAB}$ & 27,9 aA & $6,6 \mathrm{cA}$ & $6,9 \mathrm{cA}$ & 100 \\
\hline $\mathbf{T 1}$ & $1,8 \mathrm{~dB}$ & $13,6 \mathrm{bA}$ & $32,9 \mathrm{aA}$ & $12,3 \mathrm{bcA}$ & $29,2 \mathrm{aA}$ & $5,1 \mathrm{cdA}$ & $5,2 \mathrm{cdA}$ & 100 \\
\hline $\mathbf{T 2}$ & $29,8 \mathrm{aA}$ & $12,8 \mathrm{bcA}$ & $16,5 \mathrm{bB}$ & $6,2 \mathrm{cB}$ & $18,3 \mathrm{bB}$ & $7,2 \mathrm{cA}$ & $9,2 \mathrm{cA}$ & 100 \\
\hline
\end{tabular}

As médias seguidas da mesma letra minúscula na linha, para as diferentes porções da planta, e da mesma letra maiúscula na coluna, para os diferentes tratamentos, não diferem estatisticamente entre si, pelo teste Tukey, a $5 \%$ de probabilidade.

${ }^{1}$. T0: tempo zero; T1: quatro (4) dias após T0; T2: 60 dias após T1.

2/. FNov: folhas novas com menos de $10 \mathrm{~cm}$ de comprimento; FSup: folhas superiores; FMed: folhas medianas; FTrat: folha tratada; FBas: folhas basais.

. Ftrat: folha pincelada com solução de $\mathrm{H} 3 \mathrm{BO} 3$ a $10 \mathrm{mmol} \mathrm{L}^{-1}$ durante três dias consecutivos.

${ }^{4 /}$. Percentagem do boro total contido na planta. 
TABELA 2 - Massa seca (MS), teor de B e conteúdo, absoluto e percentual, de B nas folhas formadas em dois meses (entre as coletas $\mathrm{T}_{1}$ e $\mathrm{T}_{2}$ ), em mudas de abacaxi 'Pérola'.

\begin{tabular}{|c|c|c|c|c|}
\hline \multirow{2}{*}{ Folhas } & \multirow{2}{*}{ MS (g) } & \multirow{2}{*}{ Teor de B $\quad\left(\mathrm{mg} \mathrm{kg}^{-1}\right)$} & \multicolumn{2}{|c|}{ Conteúdo } \\
\hline & & & ìg $\mathrm{B}$ & $\%^{\frac{1}{1}}$ \\
\hline FNov4 $4^{2 /}$ & $0,28 \mathrm{~b}$ & $6,56 \mathrm{a}$ & $1,81 \mathrm{~b}$ & $0,95 \mathrm{c}$ \\
\hline FNov3 (24 a 26) & $1,44 \mathrm{~b}$ & $6,59 \mathrm{a}$ & $9,63 \mathrm{~b}$ & $4,79 \mathrm{~b}$ \\
\hline FNov2 (21 a 23) & $4,17 \mathrm{a}$ & $5,26 \mathrm{ab}$ & $21,8 \mathrm{a}$ & $10,88 \mathrm{a}$ \\
\hline FNov1 (17 a 20$)$ & $5,11 \mathrm{a}$ & $5,17 \mathrm{~b}$ & $26,68 \mathrm{a}$ & $13,21 \mathrm{a}$ \\
\hline
\end{tabular}

As médias seguidas da mesma letra minúscula na coluna não diferem estatisticamente entre si, pelo teste Tukey, a 5\% de probabilidade.

${ }^{1 /}$ Percentagem do boro total contido na planta.

${ }^{2 /}$ Folhas novas com menos de $10 \mathrm{~cm}$ de comprimento.

No $T_{2}$, em relação ao $T_{1}$, o teor de $B$ decresceu em todas as porções da planta, com exceção do caule. Esta redução pode ser atribuída ao efeito de diluição pelo aumento da massa seca das porções FNov, FSup e Raiz das plantas (Tabela 1), e, nas demais porções das plantas, sugere-se um efeito de redistribuição, pois não foi registrado aumento significativo da massa seca das plantas . Nesta coleta $\left(\mathrm{T}_{2}\right)$, o maior teor de $\mathrm{B}$ foi observado na FTrat.

A maior massa seca e o conteúdo de $\mathrm{B}$ mais alto (Tabela 1) foram observados na porção FMed e Fbas, no $\mathrm{T}_{0}$ e $\mathrm{T}_{1}$, e na FNov no $\mathrm{T}_{2}$, resultado já esperado, visto que o conteúdo de um nutriente é proporcional à massa seca.

Entre o $\mathrm{T}_{0}$ e o $\mathrm{T}_{1}$, ocorreu aumento significativo do conteúdo de B nas porções FMed, FTrat e FBas (Tabela 1). Na porção FTrat, este incremento é devido à aplicação foliar do $\mathrm{B}$, mas o aumento nas porções da planta adjacentes a esta folha sugere a rápida remobilização do $\mathrm{B}$ absorvido, já que a coleta no $T_{1}$ foi feita um dia após a última aplicação foliar de B.

A absorção de B, aplicado via foliar, pode ser influenciada por diferentes fatores: espécie (Goor e Lune, 1980), concentração da solução de aplicação, permeabilidade da cutícula foliar, umidade relativa do ar, idade da folha utilizada na aplicação (Shu et al., 1994) e também pelo número de vezes que a aplicação foi realizada, sucessivamente.

Considerando que, de $\mathrm{T}_{1}$ para $\mathrm{T}_{2}$, a redução do conteúdo percentual de B na FTrat foi de 50\% (Tabela 1), infere-se que ocorreu a remobilização do boro, a partir desta folha, para as demais partes da planta; e a redução do conteúdo de B de 50 e $45 \%$, nas porções FMed e FBas, respectivamente, entre o $\mathrm{T}_{1}$ e $\mathrm{T}_{2}$ e o incremento de $1485 \%$ na porção FNov (Tabela 1), sugere que parte do $\mathrm{B}$ das folhas mais velhas foi remobilizado para as folhas novas (FNov) formadas no período de 2 meses entre as duas coletas.

Nas porções formadas entre as coletas $T_{1}$ e $T_{2}$ (Tabela 2), observou-se que o teor de B é maior nas porções mais novas (FNov4 e FNov3) e que com o conteúdo ocorreu o inverso, sendo maior nas porções FNov2 e FNov1, que também apresentam a maior quantidade de MS.

Segundo Cakmak e Römheld (1997), o B, em plantas deficientes, se encontraria predominantemente na parede celular, formando complexos com substâncias pécticas. Isso poderia justificar por que o conteúdo de boro é maior nas folhas mais velhas formadas durante o experimento, ou seja, nelas o B faria parte da composição da parede celular. Em tecidos mais jovens, a parede celular ainda está em formação, logo com menor conteúdo de B.

Oertli (1994), ao estudar a distribuição desuniforme de B em plantas de tomate, também observou que o conteúdo de B acompanha a MS, ocorrendo queda da concentração devido ao efeito de diluição, mas, diferentemente do observado neste experimento, as plantas de tomate não remobilizaram o boro, não apresentando formação significativa de novos órgãos à medida que a planta se desenvolveu sem o fornecimento desse nutriente.

As plantas de abacaxi apresentam a capacidade de sintetizar manitol e sorbitol (SUGAR, 2002), logo, é provável que, pela formação de complexos manitol-B-manitol e/ou sorbitol-B-sorbitol no floema (Brown e Shelp, 1997), o B seja transportado pelo floema, justificando a formação expressiva de folhas novas e normais entre as coletas $\mathrm{T}_{1}$ e $\mathrm{T}_{2}$.

\section{CONCLUSÃO}

A formação de novas folhas, as reduções do teor e do conteúdo de $\mathrm{B}$ em folhas mais velhas, o aumento do conteúdo nas folhas mais novas e a ausência de sintomas de deficiência em plantas mantidas em solução nutritiva sem B confirmam que este micronutriente é móvel em plantas de abacaxi 'Pérola'.

\section{REFERÊNCIAS}

BERGMANN, W. Farbatlas: ernährungsstörungen bei kulturpflanzen. Jena: VEB Gustav Fischer Verlag, 1986. 306p.

BROWN, P.H.; HU, H. Phloem boron mobility in diverse plant species. Botanical Acta, v.377, p.331-335. 1998.

BROWN, P.H.; SHELP, B.J. Boron mobility in plants. Plant and Soil, Dordrecht, v.193, p. 85-101. 1997.

CAKMAK, I.; RÖMHELD, V. Boron deficiency-induced impairments of cellular functions in plants. Plant and Soil, Dordrecht, v. 193, p. 7183, 1997.

DORDAS, C.; SAH, R.; BROWN, P.H.; ZENG, Q.; HU, H. Remobilização de micronutrientes e elementos tóxicos em plantas superiores. In: FERREIRA, M.E.; CRUZ, M. C.P. da; RAIJ, B van; ABREU, C.A. de. Micronutrientes e elementos tóxicos na agricultura. Jaboticabal: Legis Summa, 2001. p. 43-70.

GOOR, B.J van.; LUNE, P van. Redistribution of potassium, boron, iron, magnesium and calcium in apples trees by an indirect method. Physiologia Plantarum, Copenhagen, v. 48, p. 21-26, 1980.

LOESCHER, W.H.; EVERARD, J.D.; CANTINI, C.; GRUMET, R. Sugar alcohol metabolism in source leaves. In: MADORE, M.A.; LUCAS, W.J. (Ed.). Carbon partitioning and source-sink interactions in plants: current topics in plant physiology. Rockville: American Society of Plant Physiologists Series, 1995. v.13, p. 170-179.

MALAVOLTA, E.; VITTI, G. C.; OLIVEIRA, S. A. de. Avaliação do estado nutricional de plantas: princípios e aplicações. Piracicaba: POTAFOS, 1997.319p.

OERTLI, J.J. Non-homogeneity of boron distribution in plants and consequences for foliar diagnosis. Communications in Soil Science and Plant Analysis, Monticello, v.25, p.1.133-1.147, 1994.

SHELP, B.J. Boron mobility and nutrition in broccoli (Brassica oleracea var. Italica). Annals of Botany, London, v.61, p.83-91, 1988

SHU, Z.H.; OBERLY, G.H.; CARY, E.E. Mobility of foliar-applied boron in one-year-old peaches as affected by environmental factors. Journal of Plant Nutrition, Monticello, v.17, n.7, p. 1.243-1.255, 1994.

SIEBENEICHLER, S.C. O Boro na cultura do abacaxizeiro 'Pérola' no norte do Estado do Rio de Janeiro. 2002. 75f. Tese (Doutorado em Produção Vegetal), Universidade Estadual do Norte Fluminense Darcy Ribeiro, Campos dos Goytacazes, Rio de Janeiro, 2002.

SUGAR Alcohols. Disponível em: <www.gnc.com/health_notes/ Food_Guide/Sugar_Alcohols. Htm>. Acesso em: 12 abril 2002. 